This is a post print accepted manuscript published in Injury 2017.48(4):903-908.

DOI:10.1016/j.injury.2017.02.007.

This document has been downloaded from TamPubutafi The Insitutional Repository of University of Tampere

\title{
Comparison of the Mini-Nutritional Assessment short and long form and serum albumin as prognostic indicators of hip fracture outcomes
}

Authors: Heli Helminen, Tiina Luukkaala, Juha Saarnio, Maria Nuotio

\section{ABSTRACT}

Introduction: Malnutrition is common among older hip fracture patients and associated with adverse outcomes. We examined M ini Nutritional Assessment short (M NA-SF) and long form (M NA-LF) and serum albumin as prognostic indicators of mobility, living arrangements and mortality after hip fracture.

M ethods: Population-based prospective data were collected on 594 hip fracture patients aged 65 and over. M NA-SF, M NA-LF and serum albumin were assessed on admission. Outcomes were poorer mobility; transfer to more assisted living accommodation and mortality one month, four months and one year post fracture. Logistic regression analyses for mobility and living arrangements with odds ratios (OR) and Cox proportional hazards model for mortality with hazard ratios (HR) and $95 \%$ confidence intervals $(\mathrm{Cl})$ were used, adjusted for age, gender, ASA grade and fracture type.

Results: All measures predicted mortality at all time-points. Risk of malnutrition and malnutrition measured by M NA-LF predicted mobility and living arrangements within four months of hip fracture. At one year, risk of malnutrition predicted mobility and malnutrition predicted living arrangements, when measured by M NA-LF. Malnutrition, but not risk thereof, measured by M NA-SF predicted living arrangements at all time-points. None of the measures predicted one-month mobility.

Conclusions: All measures were strong indicators of short- and long-term mortality after hip fracture. M NA-LF was superior in predicting mobility and living arrangements, particularly at four months. All measures were relatively poor in predicting short-term outcomes of mobility and living arrangements. 


\section{INTRODUCTION}

Protein and energy malnutrition are common among older adults (1-5), with malnutrition and risk thereof especially high in hospitalized and institutionalized elderly patients. Two-thirds of older people may be at nutritional risk or malnourished $(6,7)$. Over half of hip fracture patients have a poor nutritional status $(1,3,4,8)$. Malnutrition is acknowledged to be under-recognized and undertreated in health care (9). With widespread rising life expectancy, more elderly individuals mean more hip fractures. (10). Malnutrition contributes to falls and fractures (1). Furthermore, malnourished patients are at increased risk of perioperative complications $(4,11,12)$. M alnutrition also impairs immunity, patients' tolerance of surgical stress, wound healing, physical recovery and increases the risk of infections $(2,4,11,12)$. Complications prolong the length of hospital stay, increase readmission rates and medical costs (13). Patients' functional outcome and quality of life are impaired and the risk of mortality increases $(4,14,15)$.

Nutritional status is assessed by body mass index (BMI), calf circumference (CC), arm circumference (AC), Geriatric Nutritional Risk Index (GNRI), Malnutrition Universal Screening Tool (MUST), M ini Nutritional Assessment short (M NA-SF) and long form (M NA-LF), Nutritional Risk Screening 2002 (NRS 2002), biochemical markers like albumin and lymphocyte count and anthropometric indices. So far we have no 'gold standard` for screening nutritional risk (16).

The M NA test was validated in 1994 and subsequently used in hundreds of studies. It is a highly specific, reliable and validated nutrition screening tool for older patients in various care settings (17). MNA-SF was developed in 2000 as a screening instrument to assess nutritional status (18). It is simple, noninvasive, cheap and user-friendly. In the past three years both long and short forms of the M NA have been used to examine the association of nutritional status with outcomes in hip fracture patients. (4, 19-21). Gumiero et al. (19) established that M NA-LF is good for predicting gait status and mortality. In a study by Bell and associates (20) MNA-SF and the International Classification of Disease $10^{\text {th }}$ Revision Australian Modification (ICD10-AM ) correlated with discharge destination and mortality. Koren-Hakim et al. (21) used M NA-SF to assess in-hospital length of stay, complications, readmissions and mortality. Nuotio et al. (4) examined MNA-SF as an independent predictor of the outcomes of mobility, institutionalization and mortality.

The liver produces albumin, the most substantial plasmatic protein. Nutrition is very important for albumin levels. Cabrerizo et al. recently stated that albumin is a good marker of nutritional status in clinically stable individuals, while hypoalbuminemia predicts a prognostic factor of mortality in older patients. Age does not necessarily induce low albumin level (22). Even though M NA has been widely used and tested in various geriatric settings, only albumin is commonly used, e.g. in acute surgical care.

With worldwide population ageing more surgical in-patients are likely to be old. Hip fracture serves as a tracer condition representing old and frail acutely hospitalized patients. This population-based prospective cohort study aimed to compare nutritional status according to M NA-SF, M NA-LF and serum albumin as predictors of mobility, institutionalization and mortality outcomes at one, four and twelve months in older hip fracture patients. 


\section{MATERIAL AND M ETHODS}

\section{Study population}

Seinäjoki Central Hospital is the only hospital in the Southern Ostrobothnia providing acute surgical care. Its catchment area is approximately 200,000 and all hip fractures are treated there. The study material consists of all 634 consecutive patients aged over 65 sustaining first hip fracture between December 2011 and November 2014. Pathological or periprosthetic fractures were excluded. Five patients were excluded due to institutionalization and pre-fracture inability to walk. M NA scores were missing in 10 cases and albumin results in 35 cases, leading to exclusion. Our final comparison analyses therefore included 594 patients.

\section{Data collection and variables}

M NA-SF consists of six sections: appetite or eating problem, recent weight loss, mobility impairment, acute illness/stress, dementia or depression and body mass index. Its scores are 0-7 points malnourished, 8-11 points at risk of malnutrition and 12-14 points normal nutritional status. Patients scoring below 12 are recommended further nutritional screening with M NA-LF $(17,18)$.

M NA-LF consists of twelve more sections: living arrangements, medications, presence of pressure ulcers, quality and number of meals, fluid intake, autonomy of feeding, self-perception about health and nutrition, mid-upper arm and calf circumferences. MNA-LF scores below 17 indicate malnourished, 17-23.5 at risk of malnutrition and 24-30 normal nutritional status. (17).

American Society of Anesthesiologists (ASA) scores were used to assess general health status and severity of physical comorbidity (23) in five classes: 1 ) healthy $<65$ years, 2 ) mild systemic disease, 3) severe systemic disease, 4) severe systemic disease that is a constant threat to life and 5) moribund and not expected to survive without surgery. In our study ASA scores were categorized as 2, 3 and a combined class 4-5. A diagnosis of memory disorder was defined according to the national guideline, set by a specialist in geriatric medicine or neurology (24).

A geriatric nurse elicited baseline data from patients, their representatives or hospital staff and using the medical records during the perioperative period on the orthopedic ward. The M NA assessments were completed by the same geriatric nurse and serum albumin was also measured on admission. Median hospitalization was five days (interquartile range 3-7 days), with surgery performed on $85 \%$ of patients within $48 \mathrm{~h}$ of admission.

The follow-up data were collected by telephone interviews at one, four and twelve months after surgery by the same geriatric nurse with the same informants. All the patients or their representatives gave informed consent. The ethics committee of our hospital district approved the study design.

Prefracture, one-month, four-month and twelve-month mobility and living arrangements were elicited by similar questions. Mobility was classified as 1) ability to walk outdoors unaided, 2) ability to walk outdoors with help, indoors unaided, 3) ability to walk indoors with help and 4) inability to walk. Living arrangements were classified as 1) living independently at home, 2) living at home with organized home care, 3) living in 
assisted living accommodation and 4) living in an institution. Outcome measures of mobility and living arrangements from fracture to one, four and twelve months were defined as impaired or unchanged mobility and more supported or same living arrangements. Death dates were extracted from the hospital electronic patients' files with no losses to mortality follow-up.

\section{Statistical analysis}

Baseline data distribution including M NA-LF and albumin are presented according to M NA-SF in Table 1. Differences between normal nutrition, risk of malnutrition and malnourished were tested with Pearson's chi-square test or Fisher's exact test and skewed distributions by independent samples Kruskall-Wallis test.

Distributions of nutritional status were described by three different methods (M NA-SF, MNA-LF and albumin) at baseline and according to the follow-up outcomes of mobility, institutionalization and mortality at the chosen time-points. Logistic regression analyses for mobility and living arrangements with odds ratios (OR) and Cox proportional hazards model for mortality with hazard ratios (HR) and $95 \%$ confidence intervals $(\mathrm{Cl})$ were conducted as crude analyses and analyses adjusted for age, gender, ASA grade and fracture type. Patients immobile at baseline were excluded from mobility analyses and already institutionalized patients were excluded from living arrangements analyses. Statistical analyses were carried out with SPSS (SPSS Inc, IBM Corp, Armonk, NY, USA), Version 23.0. The p-value $<0.05$ was considered statistically significant.

Sensitivity analyses for the measures were performed using website https:// www.medcalc.org/calc/ diagnostic test.php.

\section{RESULTS}

At the time of the fracture $42(7 \%)$ patients were malnourished, $236(40 \%)$ were at risk of malnutrition and $316(53 \%)$ had normal nutritional status according to M NA-SF. According to M NA-LF 38 (7\%) patients were malnourished, $347(58 \%)$ at risk of malnutrition, 209 (35\%) had normal nutritional status and $273(46 \%)$ had albumin $<34 \mathrm{~g} / \mathrm{l}$.

Of the patients 60 (10\%), $119(20 \%)$ and $154(26 \%)$ had died one month, four months and one year after the fracture. Among the survivors, the mobility level had declined in 374 (72\%), 197 (43\%) and 79 (20\%) patients one month, four months and one year after the fracture respectively. The corresponding figures for moving into more supported living arrangements were 186 (44\%), 189 (32\%) and 157 (26\%). All nutritional measures were significantly associated with mortality at all time-points. Being at risk of malnutrition or malnourished according to MNA-LF were significantly associated with impaired mobility at four months and one year, but according to MNA-SF only being at risk of malnutrition was associated with impaired mobility. M alnutrition according to M NA-SF or M NA-LF was significantly associated with more supported living arrangements at all time-points; risk of malnutrition predicted this at four months and one year after the fracture. (Tables 2-3).

According to MNA-SF, the probability that a test result will be positive when the disease is present (sensitivity) or being at risk of malnutrition or malnourished at baseline and having declined mobility one 
year after hip fracture was 57 (95\% Cl 51-63). Sensitivity values for supported living and for mortality were $58(95 \% \mathrm{Cl} 52-64)$ and $67(95 \% \mathrm{Cl} 59-74)$.

Correspondingly, sensitivity proportions were best according to M NA-LF: 77 (95\% Cl 72-82) for impaired mobility, $76(95 \% \mathrm{Cl} 70-81)$ for supported living and $86(95 \% \mathrm{Cl} 80-91)$ for 1 year mortality. According to the albumin cut-off value of under 34, sensitivity values were poorest, $52(95 \% \mathrm{Cl} 47-58)$ for impaired mobility one year after hip fracture, $55(95 \% \mathrm{Cl} 49-62)$ for supported living and $60(95 \% \mathrm{Cl} 55-65)$ for mortality.

\section{DISCUSSION}

Our findings demonstrate that hip fracture patients' nutritional status influences outcomes like mortality, mobility level and living arrangements. Poor outcomes and death concern especially the malnourished group, but there is also a significant difference in these parameters between those at risk of malnutrition and the normal.

According to our findings M NA-SF does not detect all patients at risk of malnutrition on M NA-LF. However, M NA-SF classifies some patients as being malnourished, who on M NA-LF are at risk of malnutrition. Both instruments identify true malnutrition efficiently. If in our tests the limit for risk of malnutrition on M NA-SF had been 13 not 12, the results from MNA-SF and M NA-LF would have been very close to each other; 342 (58\%) at risk of malnutrition on M NA-SF and 347 (58\%) on M NA-LF; 210 (35\%) had normal nutritional status on M NA-SF and 209 (35\%) on M NA-LF. Nevertheless, we had acute trauma patients in our nutritional screening comparison and it may be less harmful to overestimate the nutritional risk than underestimate it. In this study low serum albumin only predicted mortality.

Few studies have compared MNA-SF and M NA-LF in community dwelling older people. MNA-SF was slightly better in one (25), M NA-LF in another (26) and the instruments were equally good in a third (27). M urphy et al. (28) tested M NA and albumin in elderly orthopedic patients and found that M NA sensitivity and specificity were comparable to albumin levels. JunDe et al. (29) compared M NA-SF, NRS2002, anthropometric measures and biochemical tests among elderly patients in a general surgery department and reported that MNA-SF may be a suitable tool for nutritional assessment. Myoung-Ha et al. (30) evaluated five different nutrition tests (M NA-LF, MNA-SF, GNRI, MUST and NRS2002) and reported that MUST is the best and that MNA-SF overestimated nutritional risk in a geriatric care hospital.

M alnutrition or risk of malnutrition is very common among old people living in institutions. In our study two thirds of institutionalized elderly patients were malnourished or at risk of malnutrition. This finding is consistent with previous reviews by Kaiser et al. and Guigoz $(6,7)$. Over half of the patients needing assistance in walking before the hip fracture were at risk of malnutrition, which also corroborates previous observations (4). ASA scores reflect pre-existing medical conditions and are higher in malnourished patients, which is likely due to a greater number of comorbidities. M emory disorders were significantly less common in patients with normal nutritional status, again corroborating previous reports $(3,4,7,14)$

Nurses are key to successful screening. M alnutrition is difficult to spot on the basis of appearance alone. Suominen et al. screened nutritional status among Finnish older patients in long-term care hospitals (1,043 patients) using MNA and BMI. MNA showed that $57 \%$ were malnourished, but the nurses' estimate was only $15 \%$. If $\mathrm{BMI}$ was $<20$ and M NA $<17,30 \%$ of elderly patients were deemed malnourished, but if BMI was $>24$ and M NA $<17$ only $2 \%$ were deemed malnourished (9). 
The strengths of this study include the large and representative population, prospective and consecutive design, and use of standardized instruments to measure nutritional status. The systemic data collection including the MNA was carried out mainly by a single nurse, which enhances the reliability of the data. Patients were not excluded from the investigation on the basis of any comorbidity, for example, memory disorder or being institutionalized.

Some limitations are also conceded. Weight and height are difficult to measure perioperatively in hip fracture patients because of pain. Consequently the nurse on the orthopedic ward estimated BMI. However, height and weight were estimated again about four months after the fracture and at that time the patients were also measured. These two BMI results were very close to each other, thus the estimates were fairly accurate. BMI was only one part of M NA and we did not study the effect of BMI separately.

$M$ any studies recommend measuring nutritional status in geriatric patients $(1,3,5-7,19,20)$, although the screening benefits remain uncertain in the Cochrane Database 2013 (16). An increase in the nutritional intake during hip fracture care and reduction in complications and improvement in the quality of life have been shown $(31,32)$. However, it was concluded in the latest Cochrane Review on nutritional supplementation for hip fracture aftercare in older people conducted in 2016 that only weak evidence for the effectiveness of protein and energy feeds on mortality and complications exists (33). It is worth noting that the methodology between the studies varied considerably.

Nutritional supplementation is included in many standardized hip fracture care programs, as also in our hospital. All hip fracture patients are advised to have the nutritional assessment and receive the same nutritional care regardless of the results of the assessment. We believe it is important to make the assessment in order to estimate the severity of the nutritional problem and also to follow up the nutritional status and the effect of the intervention over time.

M NA-SF is very simple and takes less than four minutes to administer. MNA-LF takes just over 10-15 minutes. Assessment of nutritional status is difficult on the basis of the patient's appearance. MNA tests are good assessment instruments. The tests can serve to point out the risk of malnutrition. Optimal nutrition care is just as important as exercise rehabilitation. It is important to identify malnutrition and risk of malnutrition and to take prompt actions to treat them. Similar findings are likely in other older surgical patients.

\section{CONCLUSIONS}

The short and long forms of MNA and serum albumin were strong prognostic indicators of short- and longterm mortality after hip fracture. M NA-LF was superior in predicting impaired mobility and more supportive living arrangements at four months and one year post-fracture. If the limit value for risk of malnutrition in the MNA-SF test were 13 , both MNA tests would detect malnutrition risk equally well. All measures were relatively poor in predicting short-term outcomes of mobility and living arrangements. 


\section{ACKNOWLEDGEMENTS}

We want acknowledge M s Kaisu Haanpää, RN, for her expert data collection. This study was financially supported by the Competitive Research Fund of the Hospital District of Southern Ostrobothnia and the State Research Financing of Seinäjoki Central Hospital.

\section{REFERENCES}

1. Nicholson J, Dowrick A, Liew S. Nutritional status and short-term outcome of hip arthroplasty. Journal of Orthopaedic Surgery 2012;20:331-5

2. M yung-Sang M, Sung-Soo K, Sang-Yup L, Dal-Jae J, M in-Geun Y, Sung-Sim K, Hanlim M. Preoperative Nutritional Status of the Surgical Patients in Jeju. Clinics in Orthopedic Surgery 2014;6:350-7

3. Drevet S, Bioteau C, M aziere S, Couturier P, M erloz P, Tonetti J, Gavazzi G. Prevalence of proteinenergy malnutrition in hospital patients over 75 years of age admitted for hip fracture.

Orthopaedics \& Traumatology: Surgery \& Research 100 (2014) 669-74

4. Nuotio M, Tuominen P, Luukkaala T. Association of nutritional status as measured by the MiniNutritional Assessment Short Form with changes in mobility, institutionalization and death after hip fracture. Eur J Clin Nutr. 2016;70:393-8

5. Van Bokhorst-de van der Schueren MA, Lonterman-M onasch S, de Vries OJ, Danner SA, Kramer MH, M uller M . Prevalence and determinants for malnutrition in geriatric outpatients. Clin Nutr 2013;32:1007-11

6. Kaiser MJ, Bauer JM, Rämsch C, Uter W, Guigoz Y, Cederholm T, et al. M ini Nutritional Assessment International Group. Frequency of malnutrition in older adults: a multinational perspective using the mini nutritional assessment. J Am Geriatr Soc 2010;58:1734-8

7. Guigoz Y. The M ini Nutritional Assessment (MNA) review of the literature-what does it tell us? J Nutr Health Aging 2006;10:466-85

8. Goisser S, Schrader E, Singler K, Bertsch T, Gefeller O, Biber R, et al. M alnutrition According to M ini Nutritional Assessment Is Associated With Severe Functional Impairment in Geriatric Patients Before and up to 6 M onths After Hip Fracture. J Am Med Dir Assoc 2015;16:661-7

9. Suominen $\mathrm{MH}$, Sandelin $\mathrm{E}$, Soini $\mathrm{H}$, Pitkala KH. How well do nurses recognize malnutrition in elderly patients? Eur J Clin Nutr 2009;63:292-6

10. Dhanwal D, Dennison E, Harvey N, Cooper C. Epidemiology of hip fracture: Worldwide geographic variation. Indian J Orthop 2011;45:15-22

11. Patterson BM, Cornell CN, Carbone B, Levine B, Chapman D. Protein depletion and metabolic stress in elderly patients who have a fracture of the hip. J Bone Joint Surg Am 1992;74:251-60

12. Stowers M, Lemanu D, Coleman B, Hill A, M unro J. Review Article: Perioperative care in enhanced recovery for total hip and knee arthroplasty. Journal of Orthopaedic Surgery 2014;22:383-92

13. Nikkel LE, Fox EJ, Black KP, Davis C, Andersen L, Hollenbeak CS. Impact of comorbidities on hospitalization costs following hip fracture. J Bone Joint Surg Am 2012;94:9-17

14. Nuotio M, Luukkaala T. Factors associated with changes in mobility and living arrangements in a comprehensive geriatric outpatient assessment after hip fracture. Disabil Rehabil. 2016;38:112533. 
15. Carpintero P, Caeiro JR, Carpintero R, M orales A, Silva S, M esa M. Complications of hip fracture: A review. World J Orthop 2014;5:402-11

16. Omidvari AH, Vali Y, M urray SM, Wonderling D, Rashidian A. Nutritional screening for improving professional practice for patient outcomes in hospital and primary care settings. Cochrane Database Syst Rev 2013 Jun 6;6:CD005539

17. Vellas B, Villars H, Abellan G, Soto ME, Rolland Y, Guigoz Y, et al. Overview of the M NA-Its history and challenges. J Nutr Health Aging 2006;10:456-63

18. Rubenstein L, Harker J, Salva A, Gulgoz Y, Vellas B. Screening for Undernutrition in Geriatric Practice: Developing the Short-Form M ini-Nutritional Assessment (M NA-SF). J Gerontol A Biol Sci Med Sci 2001; 56: 366-72

19. Gumieiro DN, Rafacho BP, Goncalves AF, Tanni SE, Azevedo PS, Sakane DT, et al. Mini Nutritional Assessment predicts gait status and mortality 6 months after hip fracture. Br J Nutr 2013;109:165761

20. Bell JJ, Bauer JD, Capra S, Pulle RC. Concurrent and predictive evaluation of malnutrition diagnostic measures in hip fracture inpatients: a diagnostic accuracy study. Eur J Clin Nutr 2014;68:358-62

21. Koren-Hakim T, Weiss A, Hershkovitz A, Olzrateni I, Anbar R, Gross Nevo RF, et al. Comparing the adequacy of the M NA-SF, NRS-2002 and MUST nutritional tools in assessing malnutrition in hip fracture operated elderly patients. Clin Nutr 2015 Jul 22.pii:S0261-5614(15)00193-4

22. Cabrerizo S, Cuadras D, Gomez-Busto F, Artaza-Artabe I, Marin-Ciancas F, M alafarina V. Serum albumin and health in older people: Review and meta-analysis. M aturitas 2015;81:17-27

23. Aronson WL, M cAuliffe MS, Miller K. Variability in the American Society of Anesthesiologists Physical Status Classification Scale. AANA J 2003;71:265-74

24. Suhonen J, Rahkonen T, Juva K, Pitkälä K, Voutilainen P, Erkinjuntti T. The Treatment pathway of a memory disorder patient. Duodecim 2011;127:1107-16

25. Wang J, Tsai A. The short-form mini-nutritional assessment is as effective as the full-mini nutritional assessment in predicting follow-up 4-year mortality in elderly Taiwanese. J Nutr Health Aging 2013;17:594-8

26. Kiesswetter E, Pohlhausen S, Uhlig K, Diekmann R, Lesser S, Uter W, et al.. Prognostic differences of the Mini Nutritional Assessment short form and long form in relation to 1-year functional decline and mortality in community-dwelling older adults receiving home care. J Am Geriatr Soc 2014;62:512-7

27. Simsek H, Sahin S, Ucku R, Sieber C, Meseri R, Tosun P, Akcicek F. The diagnostic accuracy of the Revised M ini Nutritional Assessment short Form for older people living in the community and nursing homes. J Nutr Health Aging 2014;18:725-9

28. Murphy M, Brooks C, New S, Lumbers M. The use of the M ini-Nutritional Assessment (MNA) tool in elderly orthopaedic patients. Eur J Clin Nutr 2000;54:555-62

29. JunDe Z, Miao W, HaiKuan W, Qiang C. Comparison of two nutrition assessment in surgical elderly inpatients in Northern China. Nutr J 2015;14:68

30. Myong-Ha B, Young-Ran $\mathrm{H}$. Evaluation of the efficacy of nutritional screening tools to predict malnutrition in the elderly at a geriatric care hospital. Nutr Res Pract 2015;9:637-43

31. Botella-Carretero JI, Iglesias B, Balsa JA, Arrieta F, Zamarron I, Vazquez C. Perioperative oral nutritional supplements in normally or mildly undernourished geriatric patients submitted to surgery for hip fracture: A randomized clinical trial. Clin Nutr 2010;29:574-9

32. Bell JJ, Bauer JD, Capra S, Pulle RC. Multidisciplinary, multi-modal nutritional care in acute hip fracture inpatients- Results of a pragmatic intervention. Clin Nutr 2014;33:1101-7 
33. Avenell A, Smith T, Curtain J, Mak J, M yint P. Nutritional supplementation for hip fracture aftercare in older people. Cochrane Database Syst Rev 2016; 
Table 1. Distribution of baseline patient characteristics according to M NA-SF ( $=594)$.

\begin{tabular}{|c|c|c|c|c|c|c|c|}
\hline \multirow[b]{2}{*}{ Women, $\mathrm{n}(\%)$} & \multicolumn{2}{|c|}{$\begin{array}{c}\text { Normal } \\
n=316(53 \%)\end{array}$} & \multicolumn{2}{|c|}{$\begin{array}{l}\text { Risk of malnutrition } \\
n=236(40 \%)\end{array}$} & \multicolumn{2}{|c|}{$\begin{array}{c}\text { Malnourished } \\
n=42(7 \%)\end{array}$} & \multirow{2}{*}{$\begin{array}{l}\text { P-value } \\
0.050\end{array}$} \\
\hline & 215 & (68) & 182 & (77) & 28 & (67) & \\
\hline Age, median (range) & 83 & $(65-97)$ & 85 & $(65-100)$ & 86 & $(65-95)$ & $\varangle .001$ \\
\hline ASA, $n(\%)$ & & & & & & & $\varangle 0.001$ \\
\hline Grade 1-2 & 68 & (22) & 19 & (8) & 2 & (5) & \\
\hline Grade 3 & 196 & (62) & 147 & (63) & 29 & (70) & \\
\hline Grade 4-5 & 51 & (16) & 69 & (29) & 10 & (24) & \\
\hline Alb, median (range) & 34 & $(20-43)$ & 33 & $(18-42)$ & 33 & $(19-42)$ & 0.066 \\
\hline BMI, median (range) & 26 & $(20-43)$ & 24 & $(15-43)$ & 21 & $(15-31)$ & $\varangle 001$ \\
\hline Fracture type, n (\%) & & & & & & & 0.224 \\
\hline Neck of femur & 192 & (61) & 141 & (59) & 28 & (67) & \\
\hline Intertrochanteric & 105 & (33) & 84 & (36) & 9 & (21) & \\
\hline Subtrochanteric & 18 & (6) & 11 & (5) & 5 & (12) & \\
\hline Pre-fracture diagnosis of memory disorder, $\mathrm{n}(\%)$ & 52 & (16) & 120 & (51) & 17 & $(40)$ & $\varangle 0.001$ \\
\hline Pre-fracture mobility, $n(\%)$ & & & & & & & $\varangle 001$ \\
\hline Independent & 229 & (73) & 64 & (27) & 7 & (17) & \\
\hline Non-independent & 85 & (27) & 172 & (73) & 35 & (83) & \\
\hline Pre-fracture living arrangement, $n(\%)$ & & & & & & & $\varangle 0.001$ \\
\hline Home & 268 & (85) & 131 & (55) & 26 & (62) & \\
\hline Other than home & 48 & (15) & 105 & (45) & 16 & (38) & \\
\hline M NA-LF points, median (range) & 24.5 & $(15.5-30)$ & 20.5 & $(15-25)$ & 15.5 & $(10.5-18.5)$ & $\varangle 0.001$ \\
\hline
\end{tabular}

Abbreviations: ASA, American Society of Anesthesiologists; M NA-SF, M ini-Nutritional Assessment short form, M NA-LF, M ini-Nutritional

Assessment long form. Missing values are not shown, but were tested and included in the percentages. Differences between groups were analysed with Pearson's chi-square test or Fisher's exact test or Kruskal-Wallis test. The $p$-value $<0.05$ was considered statistically significant. 
Table 2. Association of nutritional status measured by M NA-SF, M NA-LF and serum albumin with declined mobility 1 month, 4 months and 1 year after hip fracture.

\begin{tabular}{|c|c|c|c|c|c|c|c|c|c|c|c|c|}
\hline \multirow{3}{*}{ Follow-up time } & \multicolumn{12}{|c|}{ Declined mobility } \\
\hline & \multicolumn{4}{|c|}{1 month $(n=374, N=517)$} & \multicolumn{4}{|c|}{4 months $(n=197, N=462)$} & \multicolumn{4}{|c|}{1 year $(n=146, N=385)$} \\
\hline & $\mathrm{N}$ & $\mathrm{n}(\%)$ & OR & $(95 \% \mathrm{Cl})$ & $\mathrm{N}$ & $\mathrm{n}(\%)$ & OR & $(95 \% \mathrm{Cl})$ & $\mathrm{N}$ & $\mathrm{n}(\%)$ & OR & $(95 \% \mathrm{Cl})$ \\
\hline \multicolumn{13}{|l|}{ MNA-SF baseline } \\
\hline \multicolumn{13}{|l|}{$\overline{\text { Crude }}$} \\
\hline Normal & 289 & $217(75)$ & 1.00 & & 274 & $103(38)$ & 1.00 & & 236 & $76(32)$ & 1.00 & \\
\hline At risk of malnutrition & 196 & $132(67)$ & 0.68 & $(0.46-1.02)$ & 164 & $81(49)$ & 1.62 & $(1.09-2.40)$ & 132 & $61(46)$ & 1.81 & $(1.17-2.80)$ \\
\hline M alnourished & 32 & $25(78)$ & 1.19 & $(0.49-2.86)$ & 24 & $13(54)$ & 1.96 & $(0.85-4.54)$ & 17 & $9(53)$ & 2.37 & $(0.88-6.38)$ \\
\hline \multicolumn{13}{|l|}{ Adjusted } \\
\hline Normal & 289 & $217(75)$ & 1.00 & & 274 & $103(38)$ & 1.00 & & 236 & $76(32)$ & 1.00 & \\
\hline At risk of malnutrition & 196 & $132(67)$ & 0.51 & $(0.33-0.79)$ & 164 & $81(49)$ & 1.31 & $(0.87-1.98)$ & 132 & $61(46)$ & 1.44 & $(0.91-2.29)$ \\
\hline Malnourished & 32 & $25(78)$ & 0.98 & $(0.39-2.46)$ & 24 & $13(54)$ & 1.64 & $(0.68-3.95)$ & 17 & $9(53)$ & 1.99 & $(0.70-5.67)$ \\
\hline \multicolumn{13}{|l|}{ MNA-LF baseline } \\
\hline \multicolumn{13}{|l|}{$\overline{\text { Crude }}$} \\
\hline Normal & 200 & $141(71)$ & 1.00 & & 194 & $60(31)$ & 1.00 & & 172 & $45(27)$ & 1.00 & \\
\hline At risk of malnutrition & 290 & $214(74)$ & 1.18 & $(0.79-1.76)$ & 246 & $124(50)$ & 2.27 & $(1.53-3.37)$ & 200 & $92(46)$ & 2.33 & $(1.51-3.61)$ \\
\hline Malnourished & 27 & $19(70)$ & 0.99 & $(0.41-2.40)$ & 22 & $13(59)$ & 3.23 & (1.31-7.96) & 13 & $8(62)$ & 4.38 & $(1.36-14.1)$ \\
\hline \multicolumn{13}{|l|}{ Adjusted } \\
\hline Normal & 200 & $141(71)$ & 1.00 & & 194 & $60(31)$ & 1.00 & & 172 & $45(27)$ & 1.00 & \\
\hline At risk of malnutrition & 290 & $214(74)$ & 0.93 & $(0.60-1.45)$ & 246 & $124(50)$ & 1.84 & $(1.21-2.79)$ & 200 & $92(46)$ & 1.88 & $(1.18-2.99)$ \\
\hline Malnourished & 27 & $19(70)$ & 0.83 & $(0.32-2.09)$ & 22 & $13(59)$ & 2.40 & (0.94-6.12) & 13 & $8(62)$ & 3.28 & $(0.97-11.0)$ \\
\hline \multicolumn{13}{|l|}{ Albumin baseline } \\
\hline \multicolumn{13}{|l|}{ Crude } \\
\hline Normal 34-45 & 289 & $212(73)$ & 1.00 & & 268 & $114(43)$ & 1.00 & & 237 & $85(36)$ & 1.00 & \\
\hline $28-33$ & 182 & $128(70)$ & 0.86 & $(0.57-1.30)$ & 164 & $69(42)$ & 0.98 & $(0.66-1.45)$ & 126 & $50(40)$ & 1.18 & $(0.75-1.84)$ \\
\hline$<28$ & 46 & $34(74)$ & 1.03 & $(0.57-2.09)$ & 30 & $14(47)$ & 1.18 & $(0.55-2.52)$ & 22 & $11(50)$ & 1.79 & $(0.74-4.30)$ \\
\hline \multicolumn{13}{|l|}{ Adjusted } \\
\hline Normal 34-45 & 289 & $212(73)$ & 1.00 & & 268 & $114(43)$ & 1.00 & & 237 & $85(36)$ & 1.00 & \\
\hline $28-33$ & 182 & $128(70)$ & 0.79 & $(0.51-1.22)$ & 164 & $69(42)$ & 0.90 & $(0.59-1.36)$ & 126 & $50(40)$ & 1.16 & $(0.72-1.87)$ \\
\hline$<28$ & 34 & $34(74)$ & 0.97 & $(0.46-2.04)$ & 30 & 14 (47) & 1.04 & $(0.47-2.30)$ & 22 & $11(50)$ & 1.52 & $(0.60-3.86)$ \\
\hline
\end{tabular}

Immobile patients at baseline ( $n=9)$, mobility not known and deceased patients were excluded from analyses. $N=$ survivals. $n=n u m b e r$ of impaired mobility patients during follow-up. $\mathrm{OR}$ =incidence odds ratio, $\mathrm{Cl}=$ confidence interval. Adjusted =model was adjusted by age, sex, ASA score and fracture type. $p$-values $\mathrm{p} \varangle 0.10$ are given in bold face. 
Table 3. Association of nutritional status measured by MNA-SF, M NA-LF and serum albumin with moving to more supported living arrangements 1 month, 4 months and 1 year after hip fracture.

\begin{tabular}{|c|c|c|c|c|c|c|c|c|c|c|c|c|}
\hline \multirow{3}{*}{ Follow-up time } & \multicolumn{12}{|c|}{ M oving to more supported living arrangements } \\
\hline & \multicolumn{4}{|c|}{1 month $(n=186, N=427)$} & \multicolumn{4}{|c|}{4 months $(n=79, N=389)$} & \multicolumn{4}{|c|}{1 year $(n=90, N=334)$} \\
\hline & $\mathrm{N}$ & $\mathrm{n}(\%)$ & OR & $(95 \% \mathrm{Cl})$ & $\mathrm{N}$ & $\mathrm{n}(\%)$ & OR & $(95 \% \mathrm{Cl})$ & $\mathrm{N}$ & $\mathrm{n}(\%)$ & OR & $(95 \% \mathrm{Cl})$ \\
\hline \multicolumn{13}{|l|}{ MNA-SF baseline } \\
\hline \multicolumn{13}{|l|}{$\overline{\text { Crude }}$} \\
\hline Normal & 269 & $108(40)$ & 1.00 & & 259 & $41(16)$ & 1.00 & & 225 & $49(22)$ & 1.00 & \\
\hline At risk of malnutrition & 134 & $60(45)$ & 1.21 & $(0.80-1.84)$ & 113 & $28(28)$ & 1.75 & $(1.02-3.01)$ & 94 & $30(32)$ & 1.68 & $(0.98-2.88)$ \\
\hline Malnourished & 24 & $18(75)$ & 4.47 & $(1.72-11.6)$ & 17 & $10(59)$ & 7.60 & $(2.73-21.1)$ & 15 & $11(73)$ & 9.88 & $(3.01-32.4)$ \\
\hline \multicolumn{13}{|l|}{ Adjusted } \\
\hline Normal & 269 & $108(40)$ & 1.00 & & 259 & $41(16)$ & 1.00 & & 225 & $49(22)$ & 1.00 & \\
\hline At risk of malnutrition & 134 & $60(45)$ & 1.10 & $(0.71-1.70)$ & 113 & $28(28)$ & 1.59 & $(0.91-2.77)$ & 94 & $30(32)$ & 1.38 & $(0.79-2.43)$ \\
\hline Malnourished & 24 & $18(75)$ & 3.85 & $(1.44-10.3)$ & 17 & $10(59)$ & 8.20 & $(2.70-24.9)$ & 15 & $11(73)$ & 7.70 & (2.17-27.3) \\
\hline \multicolumn{13}{|l|}{ MNA-LF baseline } \\
\hline \multicolumn{13}{|l|}{$\overline{\text { Crude }}$} \\
\hline Normal & 196 & $78(40)$ & 1.00 & & 191 & $28(15)$ & 1.00 & & 172 & $37(22)$ & 1.00 & \\
\hline At risk of malnutrition & 211 & $95(45)$ & 1.24 & $(0.83-1.84)$ & 183 & $44(24)$ & 1.84 & $(1.09-3.12)$ & 151 & $46(31)$ & 1.60 & $(0.97-2.64)$ \\
\hline Malnourished & 20 & $13(65)$ & 2.81 & $(1.07-7.36)$ & 15 & $7(47)$ & 5.09 & $(1.71-15.2)$ & 11 & $7(64)$ & 6.39 & $(1.77-23.0)$ \\
\hline \multicolumn{13}{|l|}{ Adjusted } \\
\hline Normal & 196 & $78(40)$ & 1.00 & & 191 & $28(15)$ & 1.00 & & 172 & $37(22)$ & 1.00 & \\
\hline At risk of malnutrition & 211 & $95(45)$ & 1.12 & $(0.74-1.71)$ & 183 & $44(24)$ & 1.67 & $(0.96-2.90)$ & 151 & $46(31)$ & 1.25 & $(0.73-2.14)$ \\
\hline Malnourished & 20 & $13(65)$ & 2.43 & $(0.89-6.61)$ & 15 & $7(47)$ & 4.77 & $(1.51-15.1)$ & 11 & $7(64)$ & 4.19 & $(1.05-16.6)$ \\
\hline \multicolumn{13}{|l|}{ Albumin baseline } \\
\hline \multicolumn{13}{|l|}{$\overline{\text { Crude }}$} \\
\hline Normal 34-45 & 245 & $101(41)$ & 1.00 & & 233 & $46(20)$ & 1.00 & & 210 & $51(24)$ & 1.00 & \\
\hline $28-33$ & 145 & $66(46)$ & 1.19 & $(0.79-1.80)$ & 129 & $23(18)$ & 0.88 & $(0.51-1.54)$ & 104 & $31(30)$ & 1.32 & $(0.78-2.24)$ \\
\hline$<28$ & 37 & $19(51)$ & 1.51 & $(0.75-3.01)$ & 27 & $10(37)$ & 2.39 & $(1.03-5.57)$ & 20 & $8(40)$ & 2.08 & $(0.80-5.37)$ \\
\hline \multicolumn{13}{|l|}{ Adjusted } \\
\hline Normal 34-45 & 245 & $101(41)$ & 1.00 & & 233 & $46(20)$ & 1.00 & & 210 & $51(24)$ & 1.00 & \\
\hline $28-33$ & 145 & $66(46)$ & 1.21 & $(0.78-1.86)$ & 129 & $23(18)$ & 0.98 & $(0.55-1.74)$ & 104 & $31(30)$ & 1.85 & $(1.03-3.31)$ \\
\hline$<28$ & 37 & $19(51)$ & 1.60 & $(0.78-3.30)$ & 27 & $10(37)$ & 2.61 & $(1.07-6.33)$ & 20 & $8(40)$ & 2.11 & $(0.75-5.94)$ \\
\hline
\end{tabular}

Patients institutionalized at baseline $(n=118)$ were excluded from the analyses. Patients whose living arrangements were unknown at baseline and deceased patients were also excluded. $\mathrm{N}=$ survivals. $\mathrm{n}=$ number of patients with more supported living arrangements during follow-up. OR =incidence odds ratio, $\mathrm{Cl}=\mathrm{confidence}$ interval. Adjusted $=$ model was adjusted by age, sex, ASA score and fracture type. P-value $p<0.10$ are presented in bold face. 
Table 4. Association of nutritional status measured by MNA-SF, M NA-LF and serum albumin with mortality 1 month, 4 months and 1 year after hip fracture.

\begin{tabular}{|c|c|c|c|c|c|c|c|c|c|c|c|c|c|}
\hline \multirow{3}{*}{ Follow-up time } & \multirow[b]{3}{*}{$\mathrm{N}$} & \multicolumn{12}{|c|}{ Deceased } \\
\hline & & \multicolumn{4}{|c|}{1 month $(n=60 / N=594)$} & \multicolumn{4}{|c|}{4 months ( $n=119 / N=594)$} & \multicolumn{4}{|c|}{1 year $(n=154 / N=594)$} \\
\hline & & $\mathrm{n}$ & $(\%)$ & $\mathrm{HR}$ & $(95 \% \mathrm{Cl})$ & $\mathrm{n}$ & $(\%)$ & $\mathrm{HR}$ & $(95 \% \mathrm{Cl})$ & $\mathrm{n}$ & $(\%)$ & $H R$ & $(95 \% \mathrm{Cl})$ \\
\hline \multicolumn{14}{|l|}{ MNA-SF baseline } \\
\hline \multicolumn{14}{|l|}{$\overline{\text { Crude }}$} \\
\hline Normal & 316 & 19 & (6) & 1.00 & & 37 & (12) & 1.00 & & 51 & (16) & 1.00 & \\
\hline At risk of malnutrition & 236 & 32 & (14) & 2.29 & $(1.30-4.05)$ & 66 & (28) & 2.61 & $(1.74-3.90)$ & 82 & (35) & 2.43 & $(1.71-3.45)$ \\
\hline Malnourished & 42 & 9 & (21) & 3.84 & $(1.74-8.49)$ & 16 & (38) & 3.83 & $(2.13-6.88)$ & 21 & (50) & 3.92 & (2.35-6.51) \\
\hline \multicolumn{14}{|l|}{ Adjusted } \\
\hline Normal & 316 & 19 & (6) & 1.00 & & 37 & (12) & 1.00 & & 51 & (16) & 1.00 & \\
\hline At risk of malnutrition & 236 & 32 & (14) & 1.64 & $(0.92-2.95)$ & 66 & (28) & 1.90 & $(1.26-2.87)$ & 82 & (35) & 1.88 & $(1.32-2.69)$ \\
\hline Malnourished & 42 & 9 & (21) & 2.80 & (1.24-6.33) & 16 & (38) & 2.76 & $(1.51-5.05)$ & 21 & (50) & 2.95 & $(1.75-4.98)$ \\
\hline \multicolumn{14}{|l|}{ MNA-LF baseline } \\
\hline \multicolumn{14}{|l|}{ Crude } \\
\hline Normal & 209 & 4 & ( 2) & 1.00 & & 14 & (7) & 1.00 & & 21 & (10) & 1.00 & \\
\hline At risk of malnutrition & 347 & 46 & (13) & 7.24 & $(2.60-20.1)$ & 90 & (26) & 4.34 & $(2.47-7.62)$ & 112 & (32) & 3.77 & $(2.36-6.00)$ \\
\hline Malnourished & 38 & 10 & (26) & 15.8 & (4.94-50.3) & 15 & (40) & 7.39 & (3.57-15.3) & 21 & (55) & 7.66 & $(4.18-14.0)$ \\
\hline \multicolumn{14}{|l|}{ Adjusted } \\
\hline Normal & 209 & 4 & ( 2) & 1.00 & & 14 & (7) & 1.00 & & 21 & (10) & 1.00 & \\
\hline At risk of malnutrition & 347 & 46 & (13) & 5.03 & (1.77-14.4) & 90 & (26) & 2.92 & (1.64-5.19) & 112 & (32) & 2.73 & $(1.70-4.40)$ \\
\hline Malnourished & 38 & 10 & (26) & 10.6 & $(3.20-34.9)$ & 15 & (40) & 4.69 & $(2.23-9.86)$ & 21 & (55) & 5.11 & $(2.75-9.50)$ \\
\hline \multicolumn{14}{|l|}{ Albumin baseline } \\
\hline \multicolumn{14}{|l|}{ Crude } \\
\hline Normal 34-45 & 321 & 22 & (7) & 1.00 & & 43 & (13) & 1.00 & & 57 & (18) & 1.00 & \\
\hline $28-33$ & 221 & 33 & (15) & 2.26 & $(1.31-3.87)$ & 54 & (24) & 1.97 & $(1.32-2.94)$ & 70 & (32) & 2.01 & $(1,41-2.85)$ \\
\hline$<8$ & 52 & 5 & (10) & 1.37 & $(0.52-3.63)$ & 22 & (42) & 3.39 & $(2.03-5.67)$ & 27 & (52) & 3.46 & (2.19-5.48) \\
\hline \multicolumn{14}{|l|}{ Adjusted } \\
\hline Normal 34-45 & 321 & 22 & (7) & 1.00 & & 43 & (13) & 1.00 & & 57 & (18) & 1.00 & \\
\hline $28-33$ & 221 & 33 & (15) & 2.06 & $(1.19-3.57)$ & 54 & (24) & 1.83 & $(1.22-2.75)$ & 70 & (32) & 1.88 & $(1.31-2.68)$ \\
\hline$<8$ & 52 & 5 & (10) & 0.95 & $(0.35-2.56)$ & 22 & (42) & 2.47 & (1.46-4.18) & 27 & (52) & 2.60 & $(1.62-4.16)$ \\
\hline
\end{tabular}

